\title{
Visualizing the Geography of Platform Boundary Resources: The Case of the Global API Ecosystem
}

\author{
Jukka Huhtamäki \\ Tampere University of \\ Technology \\ jukka.huhtamaki@tut.fi \\ Martha G. Russell \\ Stanford University \\ martha.russell@stanford.edu
}

\author{
Rahul C. Basole \\ Georgia Institute of Technology \\ basole@gatech.edu
}

Marko Seppänen

Tampere University of

Technology

marko.seppanen@tut.fi

\author{
Kaisa Still \\ VTT Technical Research Centre \\ of Finland \\ kaisa.still@,vtt.fi
}

\begin{abstract}
Platform boundary resources play an increasingly transformative role in the global digital ecosystem. In this study, we focus on one type of platform boundary resource, namely application programming interfaces (APIs). Guided by two competing assumptions -1) that geographic boundaries are blurred and potentially less important in a digitally connected world, and 2) that geographic proximity matters for co-innovation-we investigate the global footprint of APIs. Using a datadriven visual network analysis approach to examine more than 15,000 APIs and mashups, we first map the global locations of where APIs are being created. We then examine how API mashups connect these locations globally and regionally. Our results show that while APIs are globally distributed, they are mainly concentrated in major entrepreneurial regions. We also find that there is a skewed distribution, with the U.S. and Silicon Valley in particular leading the way. We conclude with both theoretical and managerial implications.
\end{abstract}

\section{Introduction}

"Platform" is an increasingly popular term used in both scholarly and practitioner outlets. While several definitions exist, a platform can be broadly considered an enabler of value-creating interactions between external producers and consumers [8]. To enable seamless interactions between stakeholders, a platform provides an open, participative infrastructure and sets governance conditions. In the emerging platform economy, digital infrastructures are at the core of these value creation processes. The development of digital infrastructures, however, requires designing and managing different types of boundary resources (i.e., interfaces).

It has been argued that the true building block of this digital transformation is the rapid prominence of one particular type of boundary resource, namely application programming interfaces (APIs) [21]. APIs, along with other boundary resources such as software development kits (SDK), have been shown to transfer design capability to users, generating complementary assets in the form of applications. Over time, APIs have become an important element in governing emerging innovation networks [14]. In the context of the platform economy, these digital boundary resources have been complemented with other boundary resources, such as agreements between the platform owner and complementors pertaining to issues such as immaterial rights, sharing of benefits, and data sharing [26].

The objective of APIs is to allow others to innovate on top of digital platforms, enabling API providers to reap significant benefits from an emerging ecosystem of platforms [19]. Iver [20] has suggested that APIs are turning into the most salient form of future interfirm partnerships. More importantly, Basole [2] suggested that "as enterprises become increasingly hyperconnected, appropriately crafted API strategies will become essential to ensure survival, innovation, and growth."

At the same time, innovation, economic growth, and success have traditionally been approached as diverse elements at the global, national, regional, metropolitan, city, and individual organization level. However, recent innovation studies, following the approach of open innovation [7] and addressing the interfirm networks as complex systems characterized by co-evolving actors engaged in collaboration, take a holistic viewpoint to investigate these ecosystems of 
business [27] and innovation [30]. Iansiti and Levien [18] have highlighted the importance of relationships among these participating entities. Coupled with the digitalization of business and innovation infrastructure, resource dependencies and geo-location dependencies are changing. One example of this is the emergence and success of "born-global" companies that have not allowed geographical barriers to limit their growth [24], resulting in unicorns (defined as startups valued at $\$ 1$ billion U.S. dollars or more) emerging from unexpected locations, such as Sweden, Scotland, and the Czech Republic [6].

This paper explores the structure of the global API ecosystem. We are aware that two competing assumptions exist in this domain. On one hand, it is assumed that geographic boundaries are blurred and potentially less important in a digitally connected world. On the other hand, geographic proximity very likely still matters for open co-innovation. In order to explore these mechanisms, we investigate the global footprint of APIs. Specifically, we examine the geography of APIs by mapping and visualizing the relationships between the corporate headquarter locations offering APIs in order to better understand the global geography of co-creation and interdependency in the emerging API ecosystem. These geographical locations indicate sources of talent that have been successful in innovating a new digital product and finding a partner for it. We particularly focus on the top entrepreneurial regions identified as highly successful in nurturing startup ecosystems [9]. In doing so, we complement and extend existing digital innovation and strategy research that has investigated the overall topology of the API ecosystem [11,36] and the categorical relationships between the APIs [2]. We believe that a geographic perspective provides important new insights for the quest of supporting innovation in the context of the platform economy.

The remainder of the article is structured as follows. After the introduction, Section 2 presents related work. Section 3 describes both the methodology and the empirical context. Section 4 presents the analysis and reviews the results. Section 5 discusses the implications of our study. Concluding remarks are presented in Section 6.

\section{Related work}

In this section, we review existing work related to the exploration of geographical influences on the API infrastructure of the emerging digital platform economy. More specifically, this includes the following two viewpoints: the description of APIs as technological artifacts and the role of APIs in the larger context of digital platforms.

\subsection{APIs as technological artifacts}

The contract of one piece of computer software with another, often called an API, provides a technical artifact of the relationship between the "executable pieces of software that are offered as applications, services or systems to end-users" [14]. An API mediates between the codebases of applications and provides interoperability, extending the function of software subsystems [33] and links functionality and stakeholders to create added value.

APIs have moved to the front and center of the discussion on digital infrastructure and digital platforms over the last few years. From a technological standpoint, however, APIs have been a key part of web-centric development for more than a decade. It is important to make a distinction between the general notion of an API-something that has been routinely used in modular software development for decadesand Web APIs, boundary resources that enable thirdparty access and manipulation of data managed by an online service.

The RESTful approach for designing the architecture of individual application is at the technological core of contemporary Web development. The RESTful approach is based on Fielding's (2000) [12] dissertation work that defines a way to apply HTTP protocol to interact with resources exposed through Web APIs. Moreover, APIs are a key concept in Web 2.0 [28], a compilation of practices that transformed the Web from a page-centric collection of documents to an "architecture of participation" in which users create and share data with the help of a network of Web applications and communication with each other through APIs. For Web developers, Web APIs and the RESTful approach have introduced the means and scale to take advantage of and engineer for serendipitous reuse, effectively making the Web "an expansive application framework" [35].

Mashups are an organic derivative of APIs. Generally speaking, mashup development takes place in three complementing levels [37]. First, data can be collected from one or more sources. Even an application using an individual external source of data can be considered a mashup when it is mashing up its internal data with the external data. Second, application logic can be compiled from existing components. This is particularly true in modern Web applications thatthanks to npm, RubyGems, and other package management systems - are increasingly compositions of tens or even hundreds of existing software packages. Third, user interface can be built by using existing components, such as Google Maps, one of the early entrants to massively shared software components, for mashup development. 


\subsection{Digital platforms and APIs}

The microservices architecture that emerges from systems of APIs can be seen as a digital platform in the ecosystem of software that matches services to user needs by extracting and analyzing data, providing new forms of control relationships through monitoring and personalizing [34]. The benefits created through APIs functioning as a digital platform deliver economic value to both the companies providing the APIs and to end users of the digital services. Across multiple user groups, such API systems function as digital platforms, as they bring together multiple user groups and create positive internal network effects and network externalities $[10,23,31]$. The organizational structure of control arrangements associated with API systems as digital platforms create the boundary-setting conditions of an API ecosystem.

Weiss and Gangadharan [36] were the first to visualize the API economy. They did this with the API data from ProgrammableWeb, which they illustrated with network representations. Evans and Basole [11] showed that Amazon has built their entire business around APIs; Walmart, on the other hand, only uses a few APIs. Why is that the case? The answer is strategy. In a recent survey of global businesses, it was found that strategy, not technology, drives digital transformation [22]. Enterprises that embrace a comprehensive digital strategy and take technological risks become digital transformation leaders.

Digital platforms have further been visualized as interconnections between categories of APIs. Basole [2] has presented these interconnections as networks, establishing the emphasis on APIs in platforms. Basole stated that, "[d]ecision makers must embrace the complex relationships between the various segments to understand their position in the ecosystem and identify possible opportunities for new collaboration and innovation" [2].

\section{Methodology}

Following prior work $[2,11]$, our study uses a datadriven approach to investigate the geography of the global API ecosystem. More specifically, we conduct the investigation using data-driven visual network analytics with a process that follows the Ostinato Model $[16,17]$. The applied methodology is described from three different viewpoints. First, we give an overall description of the empirical case that is used to investigate the API ecosystem. Second, we review the specifics of the data collected for the investigation. Third, we describe the visualization process implemented to conduct the analysis. The data collection, analysis, and visualization process design follows the Ostinato Model.

\subsection{Empirical case description}

This investigation is built on an empirical case of API co-use in mashups. More specifically, we follow Weiss and Gangadharan [36], Evans and Basole [11], and Basole [2] to collect two sets of data: a collection of APIs and a collection of mashups built using one or more of the APIs. In order to create a network representation of the interconnections of individual APIs, we start from the list of mashups and connect APIs to each other on the basis of their co-use. This approach is analogous to co-citation analysis [32], which is often applied in scientometrics.

To introduce the geographical dimension to the analysis, we connect the APIs with the companies that provide them. This provides us with the home location of the API. Further, we resolve the location of each API in three different levels: the specific geolocation (latitude and longitude), the entrepreneurial region (following and extending [9]), and the global region.

We then produce a set of representations of the data. First, we examine the global distribution of API home locations. Second, we examine the interconnections between these home locations at the entrepreneurial region level. Third, we measure the internal structure of these entrepreneurial locations and investigate a few example regions in more detail.

\subsection{Data}

The data on APIs and mashups is sourced from ProgrammableWeb, the "world's leading source of news and information about Internet-based application programming interfaces (APIs)." To collect the dataset representing APIs and mashups, we implemented a tailored process that crawls the individual ProgrammableWeb pages, including API and mashup data. While ProgrammableWeb reports that some 15,000 APIs are listed, we were able to collect 13,366 APIs. This is in line with the fact that ProgrammableWeb serves the API Directory in 539 pages, each including 25 APIs, resulting in an estimated total of 13,475 APIs.

In addition to the data on APIs, we collected a total of 6,271 mashups that, in this investigation, serve as means to create connections between the APIs. Mashups listed in ProgrammableWeb are affiliated with 0 to $n$ APIs through the Related APIs property. "MIT Visual Traceroute," for example, uses "ipinfo.io" to trace the routes through which packages flow on the Internet. 
To introduce the location information to the APIs, we aggregate the API data with Crunchbase, a socially constructed dataset of some 300,000 startup and growth companies. More specifically, we use the URL information available both in the API details and the company data in Crunchbase. The URLs are reduced to their main domain, for example, google.com, to allow linking. Moreover, to manage the fact that a number of companies are using their Facebook pages, Twitter accounts, or some other repositories as their home page, we use Levenshtein distance to assist in making the connections. Levenshtein distance represents the number of editing steps needed to transform one piece of text to another. Here, we use Levenshtein distance to measure the similarity between the URL and the related company name. Specifically, we order the list of potential domains according to their distance to company name and select the closest option. We were able to resolve the location of a total of 6,218 APIs.

Further, we tap into the ISO 3166-2 Country Codes list available at Datahub ${ }^{1}$ to resolve country names. The individual locations are further categorized at two levels. First, following and extending [9], the locations are connected to city-level entrepreneurial regions, including Silicon Valley, Seattle, New York, London, and Helsinki.. Second, the country codes are used to categorize the locations into larger global regions, including North America, Europe, and East Asia \& Pacific.

For specific geolocation data, including latitude and longitude coordinates, we proceed to represent API location as city and country name pairs. For US locations, we further include the name of the state. To resolve the geolocation, we invoke geopy, a geocoding library in Python, with the textual location information.

\subsection{Visualization process}

By visualizing the network, valuable insights into the structure and dynamics of the underlying ecosystem can be gained [1]. Once the data is available locally, refined in a way that APIs and mashups can be co-referenced in a straightforward manner, and API locations are resolved, we are able to proceed to visualize the data. The visualization process follows the Ostinato Model [16]: entities to be visualized are selected, nodes and edges created, metrics calculated, layout processed, visual properties configured, and resulting networks are provisioned for the investigators for sensemaking. The process is iterated until the objectives of the investigation are met.

Custom-made processing scripts in Python were implemented for the visualization process. Several software libraries were used to support the implementation. Notable examples include Pandas for transforming and processing data in a vectorized manner and NetworkX for constructing and analyzing networks. Gephi, an interactive platform for visual network exploration, was used to create the visualizations [4].

Boundary specification is a core part of investigating an ecosystem as a network [3]. We used nodes to represent entrepreneurial regions. Nodes are connected to each other on the basis of API co-use in mashups. For the entrepreneurial regions, all possible nodes are included in the network.

Visual encoding options include node size, node color, edge weight, and network layout. In this study, node size corresponds to betweenness centrality, a well-established network metric indicating a node's role in connecting different parts of a network [13]. Betweenness centrality gives "bridge scores for boundary spanners" [15]. Edge thickness corresponds to the number of mashups connecting two entrepreneurial regions in which the company providing an API is located. In the network of entrepreneurial regions, node color reflects global regions. The network is laid out with Force Atlas, a force-driven algorithm implemented exclusively in Gephi [4].

\section{Analysis and results}

The analysis process was conducted in two stages, which are reviewed next. First, we show the geographical distribution of the home locations of the APIs according to the data. Second, we reveal the interconnections between entrepreneurial regions on the basis of API co-use in mashups.

The geographical distribution of API availability is represented in Figure 1, where the map markers indicate the home location of the APIs and the marker size corresponds to the number of APIs available in a particular location. Silicon Valley leads the field with 1,623 APIs. Second in size, companies in New York provide 459 APIs. Companies in the Seattle region, the second most important bridge in the global API ecosystem, provide a total of APIs. In Europe, London is the leading region in terms of API availability with 271 APIs.

\footnotetext{
${ }^{1}$ https://datahub.io/dataset/iso-3166-1-alpha-2-country-codes
} 


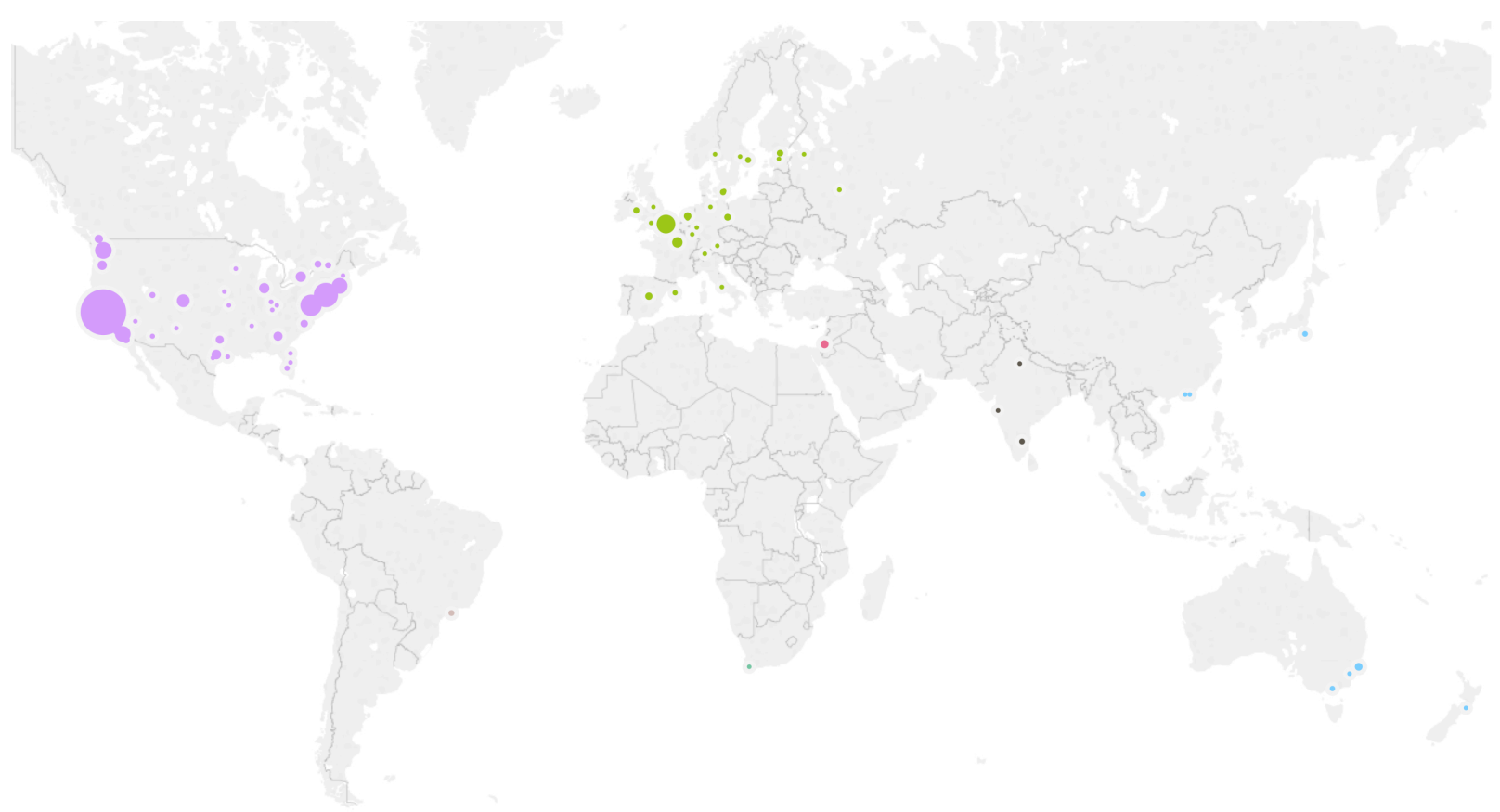

Figure 1. Global API locations

Second, we examine how the individual regions are connected through API co-use by creating a network of entrepreneurial regions as nodes that are connected to each other when APIs from the two locations are used to implement a mashup. The network visualization in Figure 2 provides additional evidence of the leading role of Silicon Valley in the global API ecosystem as the key co-location of API resources, which is connected to many other global locations. Edges are scaled according to the total number of mashups: the thicker the line, the more mashups were created using APIs from the two regions. The majority of the key entrepreneurial regions are located in North America.

To complement visual analysis with descriptive quantitative metrics, a set of measurements for the entrepreneurial regions is presented in Table 1. In addition to the number of APIs available in a region and the number of mashups using APIs from that region, we use a betweenness metric to measure the extent to which the region connects different parts of the API ecosystem. Moreover, importantly, we calculate a set of metrics for the internal structure of each of the regions. Density shows how many of the potential connections between APIs exist. Average node degree indicates the number of APIs to which an API is, on average, connected. Clustering coefficient reveals the connections in between the APIs to which a particular API is connected-a social network analogy would be the proportion of a person's friends that are also friends with each other. Moreover, we show the total number of nodes and edges in networks representing each region.

We observe that when ordered by the number of APIs available, the entrepreneurial regions roughly follow the Global Startup Index. The most notable exceptions are Tel Aviv and Berlin. Moreover, we note that just like the vast majority of the leading startup ecosystem locations, the key API resource areas are also located in North America and Europe. Tel Aviv is the only outside region in our list with a significant number of API resources, even though the global ecosystem index includes Singapore, Sao Paulo, Moscow, Bangalore, and Sydney. 
Montreal

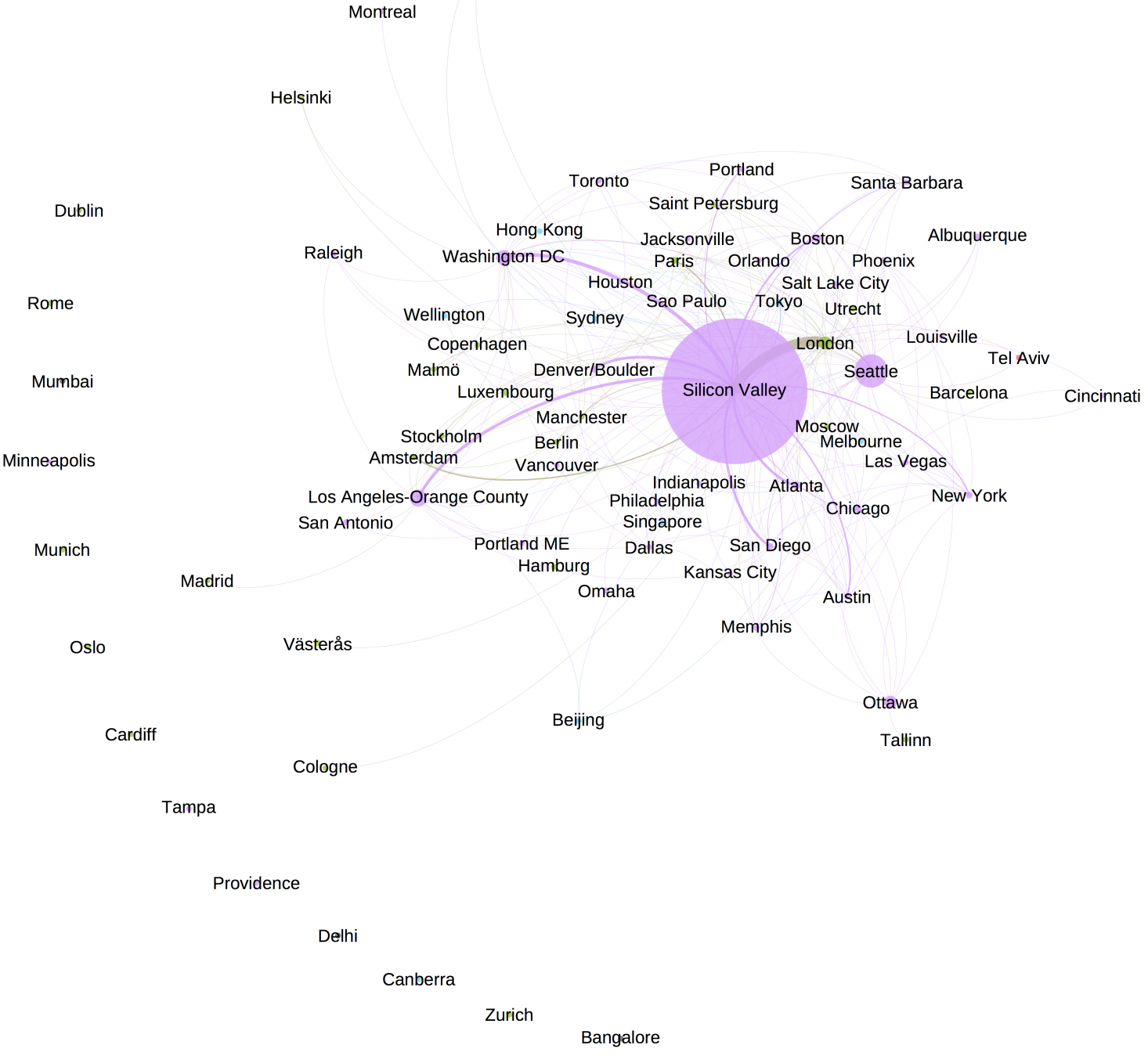

Figure 2. API co-use between entrepreneurial regions 
Table 1. Structural Characteristics of Regional API Ecosystems

\begin{tabular}{|c|c|c|c|c|c|c|c|c|c|}
\hline & $\begin{array}{l}\text { Global } \\
\text { Startup } \\
\text { Index }\end{array}$ & $\begin{array}{l}\text { API } \\
\text { Count }\end{array}$ & $\begin{array}{l}\text { Mashup } \\
\text { Count }\end{array}$ & $\begin{array}{l}\text { Region } \\
\text { Bridge } \\
\text { Score }\end{array}$ & Nodes & Edges & Density & $\begin{array}{l}\text { Average } \\
\text { Degree }\end{array}$ & $\begin{array}{l}\text { Average } \\
\text { Clustering }\end{array}$ \\
\hline Silicon Valley & 1 & 1,623 & 7,315 & 0.439 & 284 & 1,789 & 0.045 & 12.599 & 0.560 \\
\hline New York & 2 & 459 & 36 & 0.003 & 6 & 1 & 0.067 & 0.333 & 0.000 \\
\hline Washington DC & - & 353 & 121 & 0.030 & 31 & 5 & 0.011 & 0.323 & 0.097 \\
\hline London & 6 & 271 & 288 & 0.022 & 30 & 5 & 0.011 & 0.333 & 0.000 \\
\hline Seattle & 8 & 216 & 820 & 0.086 & 52 & 72 & 0.054 & 2.769 & 0.359 \\
\hline $\begin{array}{l}\text { Los Angeles- } \\
\text { Orange County }\end{array}$ & 3 & 191 & 67 & 0.035 & 21 & 3 & 0.014 & 0.286 & 0.000 \\
\hline Boston & 4 & 191 & 52 & 0.006 & 15 & 0 & 0.000 & 0.000 & 0.000 \\
\hline Denver/Boulder & - & 128 & 51 & 0.004 & 13 & 1 & 0.013 & 0.154 & 0.000 \\
\hline Paris & 11 & 85 & 28 & 0.004 & 7 & 0 & 0.000 & 0.000 & 0.000 \\
\hline Toronto & 17 & 83 & 18 & 0.000 & 7 & 0 & 0.000 & 0.000 & 0.000 \\
\hline Chicago & 7 & 82 & 27 & 0.003 & 11 & 0 & 0.000 & 0.000 & 0.000 \\
\hline Austin & 14 & 72 & 54 & 0.002 & 11 & 3 & 0.055 & 0.545 & 0.273 \\
\hline Portland & - & 67 & 37 & 0.000 & 12 & 0 & 0.000 & 0.000 & 0.000 \\
\hline Atlanta & - & 65 & 46 & 0.008 & 4 & 0 & 0.000 & 0.000 & 0.000 \\
\hline Vancouver & 18 & 54 & 3 & 0.000 & 3 & 0 & 0.000 & 0.000 & 0.000 \\
\hline Dallas & - & 51 & 8 & 0.000 & 4 & 0 & 0.000 & 0.000 & 0.000 \\
\hline Tel Aviv & 5 & 50 & 5 & 0.000 & 4 & 0 & 0.000 & 0.000 & 0.000 \\
\hline Sydney & 16 & 46 & 17 & 0.000 & 1 & 0 & 0.000 & 0.000 & 0.000 \\
\hline Amsterdam & 19 & 44 & 23 & 0.000 & 8 & 1 & 0.036 & 0.250 & 0.000 \\
\hline Raleigh & - & 43 & 10 & 0.000 & 6 & 0 & 0.000 & 0.000 & 0.000 \\
\hline Madrid & - & 42 & 2 & 0.000 & 2 & 0 & 0.000 & 0.000 & 0.000 \\
\hline San Diego & - & 40 & 51 & 0.002 & 5 & 2 & 0.200 & 0.800 & 0.000 \\
\hline Philadelphia & - & 39 & 6 & 0.000 & 2 & 0 & 0.000 & 0.000 & 0.000 \\
\hline Ottawa & - & 37 & 7 & 0.022 & 2 & 0 & 0.000 & 0.000 & 0.000 \\
\hline Berlin & 9 & 36 & 5 & 0.000 & 3 & 0 & 0.000 & 0.000 & 0.000 \\
\hline Helsinki & - & 33 & 9 & 0.000 & 3 & 0 & 0.000 & 0.000 & 0.000 \\
\hline Dublin & - & 31 & 1 & 0.000 & 1 & 0 & 0.000 & 0.000 & 0.000 \\
\hline Copenhagen & - & 30 & 2 & 0.000 & 1 & 0 & 0.000 & 0.000 & 0.000 \\
\hline Montreal & 20 & 29 & 3 & 0.000 & 3 & 0 & 0.000 & 0.000 & 0.000 \\
\hline Salt Lake City & - & 28 & 3 & 0.000 & 1 & 0 & 0.000 & 0.000 & 0.000 \\
\hline
\end{tabular}




\section{Implications}

The API ecosystem is a technical artifact of the control relationships between interacting codebases for software services. Internally and externally, value is co-created within boundary conditions established by the rules of code [25].

Our exploration of the geolocation of APIs is based on the assumption that APIs represent novel types of boundary resources, which in the context of the platform economy act as one basis of relationships between organizations. The promise of digitalization would suggest that these resources could be located anywhere. However, our research presents a clear picture of the co-location of API resources in the areas conducive for new business growth.

The API ecosystem is different from traditional platform ecosystem in terms of governance. A platform, by definition, is being governed by its owner who develops boundary resources allowing others to co-create value. Therefore, the platform owner has the means to govern the boundary resources. The developers will contribute to the development with their choices. In short, the ecosystem is coevolving [33].

However, in the API ecosystem, new services are constantly being developed as different combinations of existing APIs. If a particular API becomes too expensive to use or its boundary resources in any way become inappropriate to the developers using the API, the developers are able to start using an alternative API with a low transaction cost. For platform ecosystems, the developers are much more dependent on the platform owner.

In the API ecosystem specifically, we suggest that governance should be considered in four categories: 1) governance related to cross-country data (e.g., data residency), 2) governance related to mash-ups (in particular the combination of digital services with country restrictions), 3) governance related to technology use (e.g. restrictions placed on technologies and services in some countries, including China), and 4) governance related to API management: how to control, manage, distribute, and define APIs and their terms of service for global (public) use.

\section{Concluding remarks}

This paper makes several contributions. We contribute to the currently scarce empirical body of API research. We introduce a novel methodological approach, extending research conducted by Weiss [36], Evans and Basole [11], and Basole [2] by introducing the geographical dimension to empirical API research.
Our results support API ecosystem actors and stakeholders in policy making, API consumption, and orchestration of the API ecosystem.

The key insights for the policy makers are as follows: 1) Silicon Valley is the uncontested hub of the API economy. For example, a plethora of Google's services available as APIs are mashed up with APIs in a variety of ProgrammableWeb categories and geographical locations. 2) The Seattle region emerges as another major region, largely due to Amazon, an early entrant in the API ecosystem. 3) Google and Amazon APIs are often mashed up with APIs from Facebook, Twitter, and other consumer services. 4) In Europe, London emerges as the main API hub. However, even Europe as an aggregate is providing fewer APIs and mashups than California alone.

To explain the bias in the global distribution of API locations, we suggest that innovation with APIs requires a deep understanding and fine-tuning of code. While physical proximity of API developers to each other is not essential for development work, a shared context is absolutely vital. The co-location of development efforts has major advantages in the context setting and fine-tuning phases of development. Where the culture and mindset of transactable code is global and the user-centered design culture prevails, developers and marketers may be able to collaboratively co-create, even remotely. In situations in which the context of the platform and its users are new or changing, co-location may be helpful in reducing uncertainty and expediting development tasks.

With the assumption that the global competitive landscape in the emerging platform economy will reshape market shares, this observation strongly suggests an opportunity for European firms. We have already witnessed new global-scale distributions of profits $[5,29]$. Thus, we would encourage firms to carefully consider how they are going to participate in the API ecosystem of the platform economy competition. The geographical location of companies providing APIs provides an indicator of innovation in the digital services value chain. Since APIs in principle standardize the interfaces between participants, APIs are removing switching costs between suppliers or customers-thus remarkably changing the competition and firms' possibilities to achieve lock-in or lock-on positions. What are the first steps in entering the API ecosystem? Would it be essentially the same situation as in the app ecosystems, that is, endless competition regarding how to differentiate from the others, increase visibility, and generate scalable adoption among a sea of APIs?

This investigation has several limitations, which also describe opportunities for further research. First, 
we suggest the investigation of global patterns of API consumption, that is, the ways firms use each other's APIs to create and co-create value for their customers. However, because API consumption is considered business-critical information, representative data is not available at the system level. Second, as mentioned in discussing the implications, APIs exist beyond the source of data utilized in this investigation. An important body of APIs that is not covered in this paper consists of those providing open governmental data. On a related note, current activities around MyData are a potential source for a stream of new APIs to serve as building blocks of the API economy and true digitalization.

Several additional venues for further research exist. First, a detailed examination of the internal API co-use structure of the individual entrepreneurial regions will enable their comparison. Second, it will be interesting to observe the API-level interconnections between individual pairs of entrepreneurial regions. Third, we propose that additional data on API availability beyond company-provided resources will provide additional insights on the global API ecosystem. Lastly, we suggest conducting a global analysis of the firm-level API provision distribution and API co-use structure. We anticipate that the structure of the firm-level co-use network is scale-free, implicating that a small number of nodes play a crucial role in ensuring global connectivity.

\section{References}

[1] Basole, R.C. Visual Business Ecosystem Intelligence: Lessons from the Field. IEEE Computer Graphics and Applications 34, 5 (2014), 26-34.

[2] Basole, R.C. Accelerating Digital Transformation: Visual Insights from the API Ecosystem. IT Professional Magazine In Press, (2016).

[3] Basole, R.C., Russell, M.G., Huhtamäki, J., Rubens, N., Still, K., and Park, H. Understanding Business Ecosystem Dynamics: A Data-Driven Approach. ACM Transactions on Management Information Systems 6, 2 (2015), 32.

[4] Bastian, M., Heymann, S., and Jacomy, M. Gephi: An Open Source Software for Exploring and Manipulating Networks. Proceedings of Third International AAAI Conference on Weblogs and Social Media.(2009).

[5] Bradley, T. Android Dominates Market Share, But Apple Makes All The Money. Forbes, 2013. http://www.forbes.com/sites/tonybradley/2013/11/15/a ndroid-dominates-market-share-but-apple-makes-allthe-money/.

[6] CBInsights. The Unicorn List: Current Private Companies Valued At \$1B And Above.

cbinsights.com, 2016.

https://www.cbinsights.com/research-unicorncompanies.

[7] Chesbrough, H. The Logic of Open Innovation: Managing Intellectual Property. California

Management Review 45, 3 (2003), 33-58.

[8] Choudary, S.P., Alstyne, M.W.V., and Parker, G.G. Platform Revolution: How Networked Markets Are Transforming the Economy--And How to Make Them Work for You. W. W. Norton \& Company, New York, 2016.

[9] Compass.co. The Global Startup Ecosystem Ranking 2015. Compass.co, 2015.

[10] de Reuver, Mark, Sørensen, Carsten, and Basole, Rahul C. The Digital Platform: A research agenda. Journal of Information Technology In Press, (2016).

[11] Evans, P.C. and Basole, R.C. Revealing the API Ecosystem and Enterprise Strategy via Visual Analytics. Commun. ACM 59, 2 (2016), 26-28.

[12] Fielding, R. Architectural Styles and the Design of Network-based Software Architectures. 2000.

[13] Freeman, L.C. A Set of Measures of Centrality Based on Betweenness. Sociometry 40, 1 (1977), 35 41.

[14] Ghazawneh, A. and Henfridsson, O. Balancing platform control and external contribution in thirdparty development: the boundary resources model. Information Systems Journal 23, 2 (2013), 173-192.

[15] Hansen, D.L., Shneiderman, B., and Smith, M.A. Analyzing Social Media Networks with NodeXL:

Insights from a Connected World. Morgan Kaufmann, Burlington, MA, USA, 2011.

[16] Huhtamäki, J., Russell, M.G., Rubens, N., and Still, K. Ostinato: The exploration-automation cycle of user-centric, process-automated data-driven visual network analytics. In Transparency in Social Media. Springer, 2015, 197-222.

[17] Huhtamäki, J., Russell, M.G., and Still, K. Processing data for visual network analytics: 
innovation ecosystem experiences. In E. Bendoly and S. Clark, eds., Visual Analytics for Management Translational Science and Applications in Practice. Taylor \& Francis / Routledge, New York, 2017.

[18] Iansiti, M. and Levien, R. The Keystone Advantage: What the New Dynamics of Business Ecosystems Mean for Strategy, Innovation, and Sustainability. Harvard Business Review Press, Boston, Mass, 2004.

[19] Iyer, B. To Predict the Trajectory of the Internet of Things, Look to the Software Industry. Harvard Business Review, 2016. https://hbr.org/2016/02/topredict-the-trajectory-of-the-internet-of-things-look-tothe-software-industry.

[20] Iyer, B. and Subramaniam, M. Corporate Alliances Matter Less Thanks to APIs. Harvard Business Review, 2015. https://hbr.org/2015/06/corporate-alliances-matter-lessthanks-to-apis.

[21] Jacobson, D., Brail, G., and Woods, D. APIs: $A$ Strategy Guide. O’Reilly Media, 2011.

[22] Kane, Gerald C., Palmer, Doug, Phillips, Nguyen, Kiron, David, and Buckley, Natasha. Strategy, not Technology, Drives Digital Transformation. MIT Sloan Management Review and Deloitte University Press, 2015.

[23] Katz, M.L. and Shapiro, C. Network Externalities, Competition, and Compatibility. The American

Economic Review 75, 3 (1985), 424-440.

[24] Laanti, R., Gabrielsson, M., and Gabrielsson, P. The globalization strategies of business-to-business born global firms in the wireless technology industry. Industrial Marketing Management 36, 8 (2007), 11041117.

[25] Lessig, L. Code and Other Laws of Cyberspace. Basic Books, 1999.

[26] Marco Halén, Kari Hiekkanen, Kirsi Hyytinen, et al. Onko Suomi jäämässä alustatalouden junasta? Valtioneuvoston kanslia, 2016.
[27] Moore, J.F. Predators and prey: a new ecology of competition. Harvard Business Review 71, 3 (1993), 75-86.

[28] O'Reilly, T. What is Web 2.0: Design Patterns and Business Models for the Next Generation of Software. Communications \& Strategies, 1 (2007), 17.

[29] Ovide, S. and Wakabayashi, D. Apple's Share of Smartphone Industry's Profits Soars to 92\%. Wall Street Journal, 2015.

http://www.wsj.com/articles/apples-share-ofsmartphone-industrys-profits-soars-to-92-1436727458.

[30] Russell, M.G., Huhtamäki, J., Still, K., Rubens, N., and Basole, R.C. Relational Capital for Shared Vision in Innovation Ecosystems. Triple Helix: A Journal of University-Industry-Government Innovation and Entrepreneurship 2, 1 (2015), 36.

[31] Shapiro, C. and Varian, H.R. Information Rules: A Strategic Guide to the Network Economy. Harvard Business Review Press, Boston, Mass, 1998.

[32] Small, H. Co-citation in the scientific literature: A new measure of the relationship between two documents. Journal of the American Society for Information Science 24, 4 (1973), 265-269.

[33] Tiwana, A., Konsynski, B., and Bush, A.A. Research Commentary-Platform Evolution: Coevolution of Platform Architecture, Governance, and Environmental Dynamics. Information Systems Research 21, 4 (2010), 675-687.

[34] Varian, H.R. Computer Mediated Transactions. The American Economic Review 100, 2 (2010), 1-10.

[35] Vinoski, S. Serendipitous Reuse. Internet Computing, January/February 2008 (2008), 84-87.

[36] Weiss, M. and Gangadharan, G.R. Modeling the mashup ecosystem: structure and growth. $R \& D$ Management 40, 1 (2010), 40-49.

[37] Yu, J., Benatallah, B., Casati, F., and Daniel, F. Understanding Mashup Development. Internet Computing, IEEE 12, 5 (2008), 44-52. 